University of Nebraska - Lincoln

DigitalCommons@University of Nebraska - Lincoln

Faculty Publications: Materials Research

Science and Engineering Center

Materials Research Science and Engineering

Center

6-2007

\title{
Magnetization Reversal in Cubic Nanoparticles With Uniaxial Surface Anisotropy
}

\author{
Ralph Skomski \\ University of Nebraska at Lincoln, rskomski2@unl.edu \\ Xiao-Hui Wei \\ University of Nebraska at Lincoln \\ David J. Sellmyer \\ University of Nebraska-Lincoln, dsellmyer@unl.edu
}

Follow this and additional works at: https://digitalcommons.unl.edu/mrsecfacpubs

Part of the Materials Science and Engineering Commons

Skomski, Ralph; Wei, Xiao-Hui; and Sellmyer, David J., "Magnetization Reversal in Cubic Nanoparticles With Uniaxial Surface Anisotropy" (2007). Faculty Publications: Materials Research Science and Engineering Center. 70.

https://digitalcommons.unl.edu/mrsecfacpubs/70

This Article is brought to you for free and open access by the Materials Research Science and Engineering Center at DigitalCommons@University of Nebraska - Lincoln. It has been accepted for inclusion in Faculty Publications: Materials Research Science and Engineering Center by an authorized administrator of DigitalCommons@University of Nebraska - Lincoln. 


\title{
Magnetization Reversal in Cubic Nanoparticles With Uniaxial Surface Anisotropy
}

\author{
Ralph Skomski, Xiao-Hui Wei, and D. J. Sellmyer \\ Department of Physics and Astronomy and the Nebraska Center for Materials and Nanoscience, University of Nebraska, \\ Lincoln, NE 68588-0113 USA
}

The effect of surface anisotropy on the magnetization reversal in small magnetic particles is investigated. The model considers particles of cubic shape cut from a tetragonal crystal with cube faces in the (001) and equivalent planes. In particles having diameters of less than about $10 \mathrm{~nm}$, the coercivity approaches the Stoner-Wohlfarth limit, but the anisotropy field differs from that of the bulk of the particles. With increasing particle size, the nucleation modes acquire the character of magnetic surface or bulk modes that reduce the coercivity.

Index Terms-Coercivity, magnetic particles, nucleation, surface anisotropy.

\section{INTRODUCTION}

M AGNETIC surface anisotropy is of great importance in small-scale nanostructures such as thin films [1], [2] and nanoparticles [3], [4]. This is due to the enhanced surface-tovolume ratio and to the generally very strong second-order surface anisotropy [3]. A simple and popular surface-anisotropy model is to assume a normal or "transverse" easy axis perpendicular to the surface [5], [6], as shown in Fig. 1(a). However, this model is at odds with the spin-orbit and crystal-field origin of magnetocrystalline anisotropy. Surface anisotropy is linked to well-defined lattice planes [2], as indicated in Fig. 1(b). For example, a nanoparticle of approximately spherical shape has (1 m 0$)$ surfaces at the equator, $(00 \mathrm{n})$ surfaces at the poles, and other $(1 \mathrm{~m} \mathrm{n})$ surfaces elsewhere, each with a different surface anisotropy.

In the absence of an external field, the effect of the surface anisotropy is often small. Comparing typical surface anisotropies $K_{s}$ of about $0.5 \mathrm{~mJ} / \mathrm{m}^{2}$ [7] with domain-wall energies of order $10 \mathrm{~mJ} / \mathrm{m}^{2}$ [8], we see that the surface anisotropy is usually too weak to turn the spins in a direction favored by the surface anisotropy. In other words, the exchange between surface and bulk spins is sufficiently strong to impose the bulk behavior on the surface spins. An exception is small soft-magnetic grains with strong surface anisotropy, where the surface anisotropy dominates the bulk behavior.

Another exception is the vicinity of the nucleation field. In these cases, the leading energy contributions cancel, small corrections become important, and surface anisotropy has a big impact on the magnetization state [3], [9]. In this paper, we investigate the magnetization reversal in a magnet of cubic shape made from a magnetic material with tetragonal crystal structure. The symmetry of these particles corresponds to Fig. 1(b), with generally different lateral and top-and-bottom surface anisotropies.

\section{ORIGIN OF SURFACE ANISOTROPY}

Physically, bulk and surface anisotropies have the same origin. Aside from an often negligible magnetostatic anisotropy

Digital Object Identifier 10.1109/TMAG.2007.892869

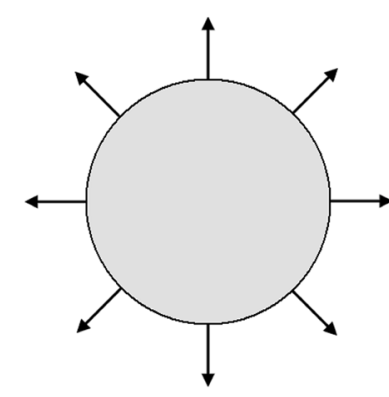

(a)

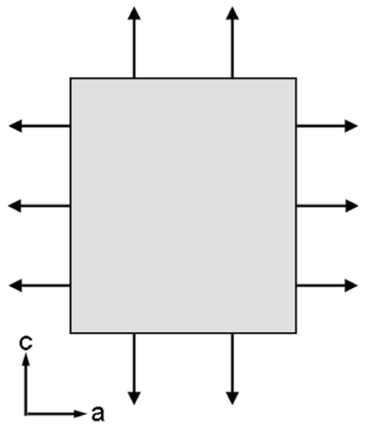

(b)
Fig. 1. Surface anisotropies: (a) unrealistic normal or "transverse" anisotropy and (b) example of a realistic scenario. The anisotropies considered in this paper are of the type (b).

contribution, magnetic anisotropy is a combined effect of spin-orbit coupling and crystal-field interaction. Spin-orbit coupling causes electrons in partially filled inner shells to develop orbital moments with aspherical charge distributions, which yield magnetic single-ion anisotropy by interacting with the anisotropic crystal field (CF). In rare-earth or $4 \mathrm{f}$ magnets, the crystal field is largely electrostatic, whereas the CF-interaction of $3 \mathrm{~d}, 4 \mathrm{~d}$, and $5 \mathrm{~d}$ electrons involves hybridization, that is, ligand fields and, in metals, bands [8], [10]. In rare-earth magnets, the spin-orbit coupling is strong and the anisotropy is determined by the relatively weak crystal field, whereas in $3 \mathrm{~d}$ transition-metal magnets, the crystal-field dominates the spin-orbit coupling and actually suppresses the orbital moment (partial quenching).

A common misunderstanding is that surface anisotropy requires an enhanced orbital moment at the surface. Essentially, surface anisotropy reflects the symmetry of the crystal field or band structure, and the orbital moment is merely a tool to realize anisotropy. A good example is rare-earth anisotropy, which is greatly enhanced by going from cubic to noncubic crystalline environments, in spite of the unchanged orbital moment of the $\mathrm{R}^{3+}$ ions responsible for the anisotropy [8]. Another counterexample is free transition-metal ions, where the orbital moment is large (unquenched) due to the absence of crystal-field charges but the anisotropy is zero by symmetry. In free-standing monatomic wires, the moment is unquenched but the axial crystal field yields a significant uniaxial anisotropy 


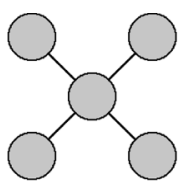

bcc (001)

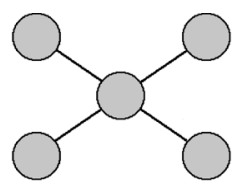

bcc (011)
Fig. 2. Surface anisotropies on a bcc surface. Both (001) and (011) surfaces yield strong second-order anisotropies with an axis normal to the plane, but only the (011) surface produces an additional second-order in-plane anisotropy due to the reduced symmetry in the film plane.

[8], [11]. These examples show that equating orbital moment and magnetic anisotropy is simplistic.

Fig. 2 illustrates how different crystalline environments at a surface yield different anisotropies. The phenomenology of surface anisotropy has been reviewed in [1], although surface anisotropy reflects single-ion magnetocrystalline anisotropy rather than Néel-type pair anisotropy. The difference is seen by considering nonmagnetic neighbors, such as $\mathrm{N}$ in $\mathrm{Sm}_{2} \mathrm{Fe}_{17}$ [8], which exhibits zero pair anisotropy but huge single-ion anisotropy.

\section{MODEL AND CALCULATION}

Our model considers particles of cubic shape and volume $L^{3}$ cut from a tetragonal crystal with cube faces in the (100) and equivalent planes. We assume that the four lateral sides have a common uniaxial second-order surface anisotropy $K_{\mathrm{ab}}$ that is generally different from the surface anisotropy $K_{\mathrm{c}}$ of the top and bottom faces. Here, the indices $a, b$, and $c$ label the directions of the surface normals. By definition, surface anisotropies $K_{\mathrm{S}}$ are anisotropy energies per area. Without loss of generality, we consider a "bulk" anisotropy of strength $K=K_{s} / D$, where $D$ is a fictitious surface thickness, and take the limit $D \rightarrow 0$ at the end of the calculation. Atomically, the anisotropy is largely confined to the surface layer, although subsurface layers affect the anisotropy by modifying the band structure.

The nucleation mode is obtained analytically, by minimizing the linearized micromagnetic free energy [3], [9]. Let us choose a coordinate frame where the $a, b$, and $c$ axes are parallel to the $x, y$, and $z$ directions, respectively. In the following figures, the $a$ and $c$ axis lie in the paper plane, as in Fig. 1, and in the remanent state, the magnetization is assumed to be in the $+z$ direction. Since $M_{\mathrm{x}}^{2}+M_{\mathrm{y}}^{2}+M_{\mathrm{z}}^{2}=M^{2}$, there are two independent magnetization components, $M_{\mathrm{x}}=m_{\mathrm{x}} M$ and $M_{\mathrm{y}}=m_{\mathrm{y}} M$. By symmetry, the considered magnetization modes are degenerate with respect to the $\pm x$ and $\pm y$ direction, and we can restrict ourselves the $m=m_{\mathrm{x}}$.

In the calculation, we must keep track of the anisotropy energy $E_{\mathrm{K}}=-K_{\mathrm{s}}(\mathbf{n} . \mathrm{M})^{2} / M_{\mathrm{S}}^{2}$, where $\mathbf{n}$ is the local easy-axis direction and $\mathbf{M}$ is the magnetization. $E_{\mathrm{K}}$ generally mixes the components $M_{\mathrm{x}}, M_{\mathrm{y}}$, and $M_{\mathrm{z}}$ of the magnetization, but the present geometry is an exception, because all faces are perpendicular to each other. Linearizing the micromagnetic free energy [3], [8] yields

$$
-A \nabla^{2} m+K_{\mathrm{p}}(x, z) m-\mu_{\mathrm{o}} M H m=0
$$

where the effective micromagnetic potential $K_{\mathrm{p}}=K_{1}$ in the bulk, $K_{\mathrm{p}}=K_{1}+K_{\mathrm{c}} / \Delta$ near the top and bottom surfaces, and $K_{\mathrm{p}}=K_{1}-K_{\mathrm{ab}} / \Delta$ near the lateral surfaces. The opposite signs of the surface contributions mean that a positive $K_{\mathrm{c}}$ and $K_{\text {ab }}$ stabilizes and destabilizes the remanent state, respectively. Note that (1) ignores shape-anisotropy effects, which is a fair approximation for sufficiently small magnets of cubic shape.

Since the $x$ - and $z$-dependent contributions to $K_{\mathrm{p}}$ are additive, the solution of (1) has the structure $m(x, z)=f_{x}(x) f_{z}(z)$. The involved functions are of the type

$$
f(\xi)=f_{\mathrm{o}} \cos (k \xi)
$$

and

$$
f(\xi)=f_{\mathrm{o}} \cosh (\kappa \xi)
$$

depending on whether the surface anisotropy a) stabilizes or b) destabilizes the original magnetization state. The two parameters in (2) obey

$$
\begin{aligned}
k^{2} & =\sqrt{\frac{\mu_{\mathrm{o}} M H_{\mathrm{N}} / 2-K_{\mathrm{p}}(x, z)}{A}} \\
\kappa^{2} & =\sqrt{\frac{K_{\mathrm{p}}(x, z)-\mu_{\mathrm{o}} M H_{\mathrm{N}} / 2}{A}}
\end{aligned}
$$

where $A$ is the exchange stiffness and $H_{\mathrm{N}}$ is the sought-for nucleation field. The mode is determined by simultaneously calculating $H_{\mathrm{N}}, k$, and $\kappa$ from (1) and (3), very similar to the calculation of nucleation modes in two-phase nanostructures [3], [9]. The calculation is subjected to the boundary conditions, $\nabla \mathbf{M}=0$ at the surface and $M=$ const. and $\nabla \mathbf{M}=$ const. at a distance $\Delta$ away from the surface. Fig. 3 shows typical spin configurations.

\section{DISCUSSION AND CONCLUSION}

There are three interesting and transparent limits. Let us first consider a relatively large soft-magnetic particle $\left(K_{1}=0\right.$ and $\left.K_{\mathrm{c}}=0\right)$ with lateral surface anisotropy $\left(K_{\mathrm{ab}}<0\right)$. In this case, $k \approx \pi / L$, and

$$
H_{\mathrm{N}}=2 A \pi^{2} / \mu_{\mathrm{o}} M L^{2}
$$

The limit is very similar to the case of a soft-magnetic inclusion in a hard matrix [9]. Equation (4) means that the surface anisotropy clamps the magnetization at the surface but does not enter the expression for the nucleation field (coercivity).

As a second example, we consider a large particle with easyaxis anisotropy $\left(K_{1}>0\right.$ and $\left.K_{\mathrm{c}}=0\right)$ where the lateral surface anisotropy facilitates nucleation $\left(K_{\mathrm{ab}}>0\right)$, roughly corresponding to Fig. 3(a). In this case, evaluation of (1) yields in lowest order

$$
H_{\mathrm{N}}=\left(2 K_{1} / \mu_{\mathrm{o}} M\right)\left(1-K_{\mathrm{ab}}^{2} / A K_{1}\right) .
$$

As expected, the coercivity decreases due to surface anisotropy. Since the domain-wall energy $\gamma=4\left(A K_{1}\right)^{1 / 2}$, the small parameter of the approximation is the ratio of surface anisotropy 
(a)

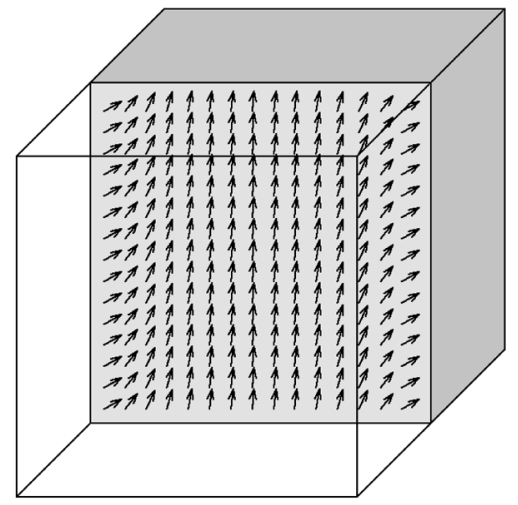

(b)

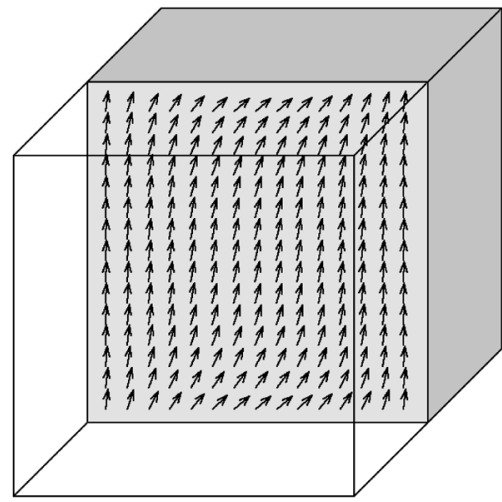

(c)

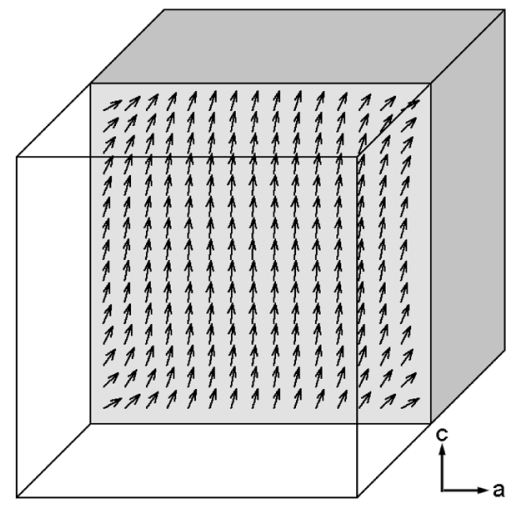

Fig. 3. Nucleation modes in the presence of surface anisotropy: (a) $K_{\mathrm{ab}}>0$ and $K_{\mathrm{c}}=0$, (b) $K_{\mathrm{ab}}<0$ and $K_{\mathrm{c}}<0$, and (c) $\mathrm{K}_{\mathrm{ab}}>0$ and $\mathrm{K}_{\mathrm{c}}<0$. In (a), the magnetization deviation $\mathbf{m}=\mathbf{M}-M \mathbf{e}_{z}$ decays exponentially in the bulk, with a field-dependent decay length.

to domain-wall energy, $K_{\mathrm{ab}} / \gamma$. Taking $K_{\mathrm{ab}}=0.5 \mathrm{~mJ} / \mathrm{m}^{2}$ and $\gamma=10 \mathrm{~mJ} / \mathrm{m}^{2}$ for a typical semihard magnet we obtain a surface correction of $4 \%$. For hard magnets, the correction is negligible $(<1 \%)$, but for soft magnets it is large and higher-order terms must be considered in (5). Expressions similar to (5) exist for other cases. For example, $K_{\mathrm{ab}}<0$ and/or $K_{\mathrm{c}}>0$ enhance $H_{\mathrm{N}}$.
Third, for very small particles, the modes become nearly coherent and

$$
H_{\mathrm{N}}=\frac{1}{\mu_{\mathrm{o}} M}\left(K_{1}+2\left(K_{\mathrm{c}}-K_{\mathrm{ab}}\right) / L\right) .
$$

This is a Stoner-Wohlfarth (SW) nucleation field with a renormalized anisotropy. The transition to $\mathrm{SW}$ behavior is similar to that in other nanostructures [3], [9] and depends on $K_{\mathrm{ab}}, K_{\mathrm{c}}$, and $A$. As a rough estimate, it occurs for particle sizes of order $10 \mathrm{~nm}$.

In conclusion, we have investigated the influence of surface anisotropy on the magnetization reversal in small magnetic particles of cubic shape. The effect depends on the lattice planes that form the surfaces and leads to various spin configurations. Using an effective micromagnetic potential, we have shown that the surface effect is often small but enhanced near the nucleation field. For very small particles, the nucleation mode is Stoner-Wohlfarth like, whereas for large particles, there exist different scenarios, depending on the bulk and surface anisotropies.

\section{ACKNOWLEDGMENT}

This work was supported in part by the National Science Foundation MRSEC, DOE, INSIC, and NCMN.

\section{REFERENCES}

[1] D. S. Chuang, C. A. Ballantine, and R. C. O'Handley, "Surface and step magnetic anisotropy," Phys. Rev. B, vol. 49, pp. 15084-15095, 1994.

[2] D. Sander, R. Skomski, C. Schmidthals, A. Enders, and J. Kirschner, "Film stress and domain wall pinning in sesquilayer iron films on W(110)," Phys. Rev. Lett., vol. 77, pp. 2566-2569, 1996.

[3] R. Skomski, "Nanomagnetics," J. Phys.: Condens. Matter, vol. 15, pp. R841-896, 2003.

[4] M. Jamet, W. Wernsdorfer, C. Thirion, D. Mailly, V. Dupuis, P. Mélinon, and A. Pérez, "Magnetic anisotropy of a single cobalt nanocluster," Phys. Rev. Lett., vol. 86, pp. 4676-4679, 2001.

[5] A. Aharoni, Introduction to the Theory of Ferromagnetism. Oxford, U.K.: Oxford Univ. Press, 1996.

[6] H. Kachkachi and E. Bonet, "Surface-induced cubic anisotropy in nanomagnets," Phys. Rev. B, vol. 73, pp. 224402-1-224402-7, 2006.

[7] U. Gradmann, "Magnetism in ultrathin transition metal films," in Handbook of Magnetic Materials, K. H. J. Buschow, Ed. Amsterdam, The Netherlands: Elsevier, 1993, vol. 7, pp. 1-95.

[8] R. Skomski and J. M. D. Coey, Permanent Magnetism. Bristol, U.K.: IOP, 1999.

[9] R. Skomski and J. M. D. Coey, "Giant energy product in nanostructured two-phase magnets," Phys. Rev. B, vol. 48, pp. 15812-15816, 1993.

[10] C. J. Ballhausen, Ligand Field Theory. New York: McGraw-Hill, 1962.

[11] M. Komelj, C. Ederer, J. W. Davenport, and M. Fähnle, "From the bulk to monatomic wires: An Ab initio study of magnetism in Co systems with various dimensionality," Phys. Rev. B, vol. 66, p. 140407, 2002.

Manuscript received October 31, 2006 (e-mail: rskomski@neb.rr.com). 